\title{
Micro RNAs and the biological clock: a target for diseases associated with a loss of circadian regulation
}

\author{
Qianwen $\mathrm{Ma}^{1,2}$, Genlin $\mathrm{Mo}^{3}$, Yong Tan ${ }^{2}$
}

1. Gynecology department, Zhenjiang Hospital Affiliated to Nanjing University of Chinese Medicine (Zhenjiang Hospital of Traditional Chinese Medicine), Zhenjiang, China.

2. Reproductive medicine department, Affiliated Hospital of Nanjing University of Chinese Medicine, Nanjing, China.

3. Advanced manufacturing institution, Jiangsu University, Zhenjiang, China.

\begin{abstract}
Background: Circadian clocks are self-sustaining oscillators that coordinate behavior and physiology over a 24 hour period, achieving time-dependent homeostasis with the external environment. The molecular clocks driving circadian rhythmic changes are based on intertwined transcriptional/translational feedback loops that combine with a range of environmental and metabolic stimuli to generate daily internal programing. Understanding how biological rhythms are generated throughout the body and the reasons for their dysregulation can provide avenues for temporally directed therapeutics.

Summary: In recent years, microRNAs have been shown to play important roles in the regulation of the circadian clock, particularly in Drosophila, but also in some small animal and human studies. This review will summarize our current understanding of the role of miRNAs during clock regulation, with a particular focus on the control of clock regulated gene expression.
\end{abstract}

Keywords: MicroRNAs; biological clock; circadian rhythm.

DOI: https://dx.doi.org/10.4314/ahs.v20i4.46

Cite as: $M a Q, M o G$, Tan Y. Micro RNAs and the biological clock: a target for diseases associated with a loss of circadian regulation. Afri Health Sci. 2020;20(4):1887-94. https:// dx.doi.org/10.4314/abs.v20i4.46

\section{Introduction}

Circadian clock represents a ubiquitous internal mechanism that allows organisms to adapt to cyclic changes in temperature, light, and other environmental factors ${ }^{1}$. Circadian clock is a 24 hour cycle that is generated endogenously, and can be modulated by external cues including natural light and temperature ${ }^{2}$. Circadian clock determines the sleeping and feeding patterns of all mammals, including humans ${ }^{3}$. Accumulating evidence demonstrates that the disruption of normal circadian clock contributes to the progression of cardiovascular disease ${ }^{4}$, metabolic disorders ${ }^{5-6}$, chemoresistance ${ }^{7}$,

\footnotetext{
Corresponding author:

Yong Tan,

Affiliated Hospital of Nanjing University

of Chinese Medicine, Number 155,

Hanzhong Road, Gu lou District, Nanjing,

Jiangsu, China, 210000.

Tel: 025-86617141; Fax: 025-86617141

E-mail: nzyszy@163.com
}

and neurodegenerative diseases ${ }^{8}$. Several mechanisms of functional impairment due to an abnormal circadian clock have been proposed, including altered cell signaling, cellular metabolic changes, and inflammation6. Scientists has revealed mechanisms underlying circadian clock function through studying Drosophila. As a model organism, the study of circadian rhythm in drosophila was initiated in 1971 when scientists obtained the first mutant of the clock Per in Drosophila through the screening of chemical mutagens. This landmark study opened up a new era for the study of molecular mechanisms of circadian rhythm. Rhythmic behavior could be evaluated to the function of several clock genes that produce circadian oscillations in certain brain neurons, which finally adjust the behavior in a circadian manner'. On a molecular level, circadian rhythm (CR) is driven by a series of transcriptional and translational feedback loops that drive the cyclic expression of clock genes including clock and bmal1, per1-3 and cry1-2, as well as their translational products a BMAL1-CLOCK complex, PER1-3, and CRY12. The levels of these clock genes serve as markers of $\mathrm{CR} 8,{ }^{1011}$. The suprachiasmatic nucleus $(\mathrm{SCN})$, a region

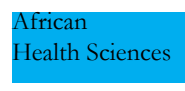

(C) 2020 Ma Q et al. Licensee African Health Sciences. This is an Open Access article distributed under the terms of the Creative commons Attribution License (https://creativecommons.org/licenses/BY/4.0), which permits unrestricted use, distribution, and reproduction in any medium, provided the original work is properly cited. 
of the hypothalamus situated above the optic chiasm, is the core circadian clock $^{12}$. The peripheral clocks are biological oscillators which can specifically regulate various physiological activities in different tissues by regulating the expression of downstream clock genes ${ }^{13}$. It is now recognized that many molecular mechanisms influence circadian gene expression including changes to the architecture of chromatin ${ }^{14}$, interactions with transcription factors ${ }^{10}$, post-transcriptional RNA modifications ${ }^{3}$, post-translational modifications ${ }^{15-16}$, and protein trafficking and degradation ${ }^{7,17-18}$. Circadian rhythm is a positive and negative feedback loop composed of major clock genes, such as Clock, Bmal1, Per and Cry. Clock and Bmall transcription factors belong to the positive feedback loop, while Per and Cry transcription factors belong to the negative feedback loop. The positive feedback loop promotes transcription of clock genes. The negative feedback loop inhibits transcription of clock genes ${ }^{19}$. Recently, alternative RNA splicing and microRNAs (miRNAs) have emerged as key players in clock regulation, raising the intriguing possibility that clock-controlled miRNAs contribute to disorders of the human circadian system. In this review, we provide an up-to-date overview of the role of miRNAs in circadian clock physiology, and discuss new methods to harness their ability to influence circadian physiology.

\section{MicroRNA discovery and synthesis}

miRNAs are non-coding RNAs consisting of 21-25 nucleotides that act as regulators of gene expression in eukaryotic cells. In eukaryotes, miRNAs are synthesized from primary miRNAs in a 2 -stage process by the action of two RNase III-type proteins termed Drosha, in the nucleus, and Dicer, in the cytoplasm. In recent years, significant advances have been made in our knowledge of miRNA processing in mammalian cells. The process is initiated when the genes for miRNAs are transcribed to a primary miRNA by RNA polymerase II. The primary miRNA is then processed in the nucleus to a precursor miRNA (pre-miRNA) of $70-100$ nucleotides by Drosha. The pre-miRNA is exported to the cytoplasm via exportin-5 and further processed by Dicer into mature miRNAs. These are then loaded onto the Argonaute protein to produce the effector RNA-induced silencing complex (RISC).

miRNAs were first discovered in 1993 by Lee and co-workers in experiments utilizing the embryos of the nematode Caenorhabditis elegans (C. elegans) ${ }^{20}$. In these organisms, the downregulation of LIN-14 is es- sential to the progression of the first larval stage L1 to L2. LIN-14 downregulation was shown to depend on the transcription of a secondary gene termed lin-4. It was found that the transcribed lin-4 was not translated into a protein, but instead gave rise to two small RNAs (21 and 61 nucleotides long), the longer sequence forming a stem-loop and acting as a precursor for the shorter RNA. Later, the same group along with Wightman ${ }^{21}$ discovered that the smaller RNA had antisense complementarity to the 3'-UTR of lin-14 mRNA. This binding decreased LIN-14 expression at the protein level without influencing its mRNA abundance. These two studies together proposed the now accepted model wherein base pairing between multiple lin- 4 miRNAs to complementary sites in the 3'-UTR of lin-14 mRNA leads to translational suppression, promoting the progression from L1 to L220-21. In 2000, it was discovered that a second miRNA, let-7, was required for the development of a later larval stage in C. elegans ${ }^{22}$. Importantly, homologues of this gene were then discovered in many organisms including human cells, revealing cross-species roles for miRNAs. In the period that followed, a large number of studies from multiple laboratories cloned miRNAs from humans, worms, and flies. The miRNAs share many features; they are 19 to 24 nucleotides in length, non-coding, and derive from a longer precursor with a stem-loop structure. Most of the identified miRNAs are evolutionarily conserved and display cell-type specificity ${ }^{23}$. To-date, multiple miRNAs have been discovered across numerous species of plants and animals. However, their biological significance often remains poorly defined and requires functional validation.

\section{miRNAs and their functional relevance}

The importance of miRNAs is highlighted by the fact that animals which fail to express them do not survive ${ }^{24}$. Dicer silencing is the accepted method of miRNA inhibition, and leads to embryonic lethality in mice due to abnormal morphology of almost all organs ${ }^{25}$. The differentiation of embryonic stem cells (ESCs) is key to organ development and is modulated by miRNAs. In mice, Dicer silencing does not influence the formation of ESC colonies, but severely impairs their differentiation $^{25-26}$. MiR-290 clusters that are expressed from a single transcript directly target the cell cycle suppressors p21 and LATS2, facilitating ESC G1-S phase progres$\operatorname{sion}^{27-28}$. The transcription factors Oct3/4, Nanog, and $\mathrm{Sox}^{2}$, are required for the maintenance of ESC pluripotency and bind to the promoter of miR-290 clusters in order to sustain their expression, thereby promoting 
self-renewal and maintaining pluripotency. In contrast, miRNA let-7 is a suppressor of pluripotency and inhibits $\mathrm{miR}-290^{29}$. In the differentiated state, let-7 is upregulated suggesting that its antagonistic effects promote ESC differentiation. miRNAs similarly regulate hematopoietic stem cells. MiR-125b is a miRNA that regulates inflammation and innate immunity by specifically promoting macrophage differentiation and activation through nuclear factor (NF)- $x \mathrm{~B}$ pathway signaling ${ }^{30-32}$. Accordingly, the dysregulation of miR-125b has been reported in many cancers, including leukemia.

miRNAs have now been shown to play a major role in an array of cell types. Although the miRNA class of molecules is ubiquitously expressed, their specific spatial and temporal expression is regulated according to tissue type. Examples include the requirement for miR273 during neuronal development ${ }^{33}$, the role of miR-1 in cardiogenesis by regulating Hand ${ }^{2}$ expression ${ }^{34}$, miR133a and miR-27 that regulate myocyte proliferation ${ }^{34-35}$, miR-203 that is induced during stratification of mouse skin and controls basal to suprabasal transition ${ }^{36}$, miRNA-375 that regulates pancreatic development ${ }^{37}$, miRNA-430 that participates in neuronal development in zebrafish $^{38}$, and miRNA-181b that inhibits IGF-1R expression and can repress glioma development ${ }^{39}$. These and emerging studies have unequivocally established the relevance of miRNAs to mammalian development and the regulation of important physiological processes.

Bahk YC40 showed a close correlation between biorhythm and suicidal ideation in patients with depression through investigation. The occurrence of suicidal thoughts in patients with morning depression was significantly less than that in patients with night depression. La Morgia ${ }^{41}$ also studied that the occurrence of biorhythm disorder is closely related to the occurrence and development of alzheimer's disease. To sum up, the disturbance of biological rhythm will cause the dysfunction of the body's nervous system, which can lead to the occurrence and development of a variety of neurological diseases.

\section{miRNAs and the control of circadian rhythms Drosophila studies}

CR processes are driven by endogenous molecular clocks that regulate the expression of clock-controlled genes (CCGs). The transcription of CCGs is controlled by the rhythmic action of transcription factors and circadian alterations in chromatin ${ }^{14,42}$. In recent years, the importance of post-transcriptional regulation which al- ters the levels and phase regulation of CCG mRNA and protein expression in CR has emerged ${ }^{3,33,42-45}$.

Circadian clocks enable organisms to adapt to fluctuating environmental conditions. In this regard Garaulet and colleagues ${ }^{46}$ found that miR-124 could regulate the CR of Drosophila. During normal light/dark cycles, miR-124 loss-of-function mutations caused abnormal locomotor activity, leading to a loss of anticipatory capacity during morning/evening transitions. In addition, miR-124 mutants caused behavioral alterations in continual darkness. Anatomical and functional tests revealed a normal circadian pacemaker in miR-124 mutants, suggesting that miR-124 regulates clock output. After profiling the miR-124 interaction network, targets in the Bone Morphogenetic Protein (BMP) pathway were shown to correct the evening activity phase shift in continual darkness. It was therefore confirmed that BMP signaling drives specific circadian behaviors in miR-124 knock-out flies.

Chen ${ }^{47}$ also studied Drosophila of circadian rhythms. They found that mRNA-276a regulates molecular and behavioral rhythms by inhibiting expression of important CCGs. Dysregulation of miR-276a in clock neurons altered timeless expression and increased arrhythmicity in constant darkness. MiR-276a was shown to be light-regulated and its expression controlled by the transcriptional activator Chorion factor 2 (CF-2). Deletion of the miR-276a-binding sites in the timeless 3'-UTR led to elevated levels of timeless protein expression and enhanced arrhythmicity. Thus, this pathway contributes to more robust rhythms observd under light/dark conditions compared to constant darkness.

Sun $^{48}$ continued to study Drosophila. They found that high levels of miRNA-279 and miRNA-996 in Drosophila regulated multiple biosynthetic processes. miRNA-279 deficiency could limit the synthesis of CO2 sensory neurons and regulate CR. miRNA-996 was found to localize adjacently to miR-279 and possess similar functionality.

Cusumano $^{49}$ also found there was relationship between Drosophila and circadian rhythms. They revealed the role of miR-210 in regulating circadian rhythm outputs and guiding/remodeling PDF positive $\mathrm{LNv}$ branches in Drosophila, and suggested that miR-210 may have pleiotropic effects on the clock, light perception and neuron development. 
Moreover, You ${ }^{50}$ studied Drosophila of biological rhythms. They founded miR-263b and miR-274 in detail and found that they both have the function of regulating behavior in adult astrocytes. Astrocyte-specific inhibition of miR-263b or miR-274 in adults significantly impaired circadian rhythm, but had no effect on the viability of glial or clock neuron cells. Knockdown of glial cells in two hypothetical miR-274 targets, CG4328 and MESK2, resulted in a significant decrease in rhythmicity. Homology between target genes of miR-274 and mammals suggests that its mechanism may be related to glial regulation of rhythms.

\section{Rat and mouse models}

Although progress in the field had implicated miRNAs in development and disease, the expression and function of miRNAs in the nervous system was not as well characterized. In 2007, Chen and colleagues ${ }^{51}$ examined two brain-specific miRNAs located in the SCN, miR-219 and miR-132, for their ability to modulate CR. Their studies revealed that miR-219 was a target of the Clock/Bmall complex which exhibited CR dependent expression, and that in vivo knockdown of miR-219 lengthened the circadian period. They further showed that miR-132 was induced by photic cues that are dependent on the MAPK/CREB-signaling axis, which was found to modulate CCG expression, and attenuate the entraining effects of light. To address the mechanisms underlying these effects, they compiled a list of targets of miR-132 and miR-219 using prediction algorithms and speculated that miR-219 and/or miR-132 could influence the clock via alterations in cell excitability. When cultured cortical neurons were transfected with miR-219 or miR-132 and the effects on depolarization- and glutamate receptor-evoked Ca2+ responses assessed, miR-132 significantly potentiated $\mathrm{K}+$, glutamate, and NMDA administration responsiveness, whilst miR-219 triggered a modest but significant attenuation of evoked responsiveness. Collectively, this study revealed that both light-responsive miR-132 and clock-regulated miR-219 influence membrane potential and cellular excitability.

Shende ${ }^{52}$ examined miR-142-3p for evidence of circadian expression in the SCN and regulation of its putative CCG target Bmal1 via specific binding sites in the 3' UTR. They also assessed miR-142-3p overexpression-induced changes in the CR of Bmal1. Mutagenesis studies revealed that two independent miRNA recognition elements equally contributed to miR-142-3p-in- duced repression. Overexpression of miR-142-3p also abolished circadian variation and endogenous Bmal1 protein levels in vitro. This confirmed that miR-142$3 p$ contributes to post-transcriptional modulation of Bmal1 and its contribution to CR.

Furthermore, Ding ${ }^{53}$ investigated the mechanisms of post-transcriptional regulation of CCGs through screening changes in miRNA levels in the pineal gland. miR-182 was found to target the 3'-UTR of clock and regulated Clock expression following hypoxic exposure of cultured pinealocytes. Zhang and colleagues 47 found that miR-27b-3p exhibits rhythmic expression in the metabolic tissue of mice exposed to constant darkness. The expression of miR-27b-3p was induced in the livers of unfed and ob/ob mice and the oscillatory expression of miR-27b-3p could be reversed by restricted feeding, suggesting its contribution to regulation of the peripheral clock. The same study identifiedmal1 as a direct target of miR27b-3p explaining its role in CR and energy metabolism in the liver.

MiR-155 was also found to regulate biological rhythm via bmal1. In bone marrow, Bmal1 could inhibit the activation of $\mathrm{NF}-x \mathrm{~B}$ and inhibit the synthesis of miR155 , allowing rats to avoid septicopyemia in response to Lipopolysaccharide (LPS). Two miR-155 binding sites were identified in the 3'-UTR of Bmall and miR-155 binding inhibited Bmal1 expression. Cells lacking miR155 had changes in CR and altered synthesis of an array of cellular factors that control the biological clock ${ }^{54}$.

Gao55 also studied circadian rhythms in the mouse models. They found that miR-17-5p, which had been previously implicated in tumor biology, also controlled CR. miR-17-5p was shown to be rhythmically expressed in synchronized fibroblasts and mouse SCN and inhibited the translation of clock by binding to its 3'-UTR. Clock also directly bound to the miR-17 promoter and enhanced its transcription and production suggesting a reciprocal relationship. Changes in Clock expression led to increased Cry1 expression and shortening of the free-running period in in vivo studies. Thus miR-17-5p was shown to play an important role in stabilization of the circadian-clock through indirect regulation of Cry1. In addition, Kiessling ${ }^{56}$ also revealed a strain and light-specific function of miR-132/212 in the circadian mechanism, suggesting that miR-132 and miR-212 are background-dependent circadian regulators. 
Moreover, Yoo $^{57}$ also found that Bmall mRNA and protein oscillation amplitude as well as Cry1 protein oscillation increased in Per2::LucSV mice, suggesting that rhythmic overexpression of Per2 could enhance the expression of core clock genes such as Per2.

\section{Human studies}

$\mathrm{Li}^{58}$ identified that clock expression levels were elevated in high grade glioma tissue compared to low grade glioma and normal tissue. miR-124 was found to directly act upon clock and inhibit its expression. In glioma, the downregulation of miR-124 led to increased clock expression and reduced proliferation and migration of glioma cells. Clock was further found to regulate glioma cell proliferation and migration through NF-xB signaling ${ }^{58}$.

In addition, $\operatorname{Han}^{59}$ found that miR-34a reduced the likelihood of cholangiocarcinoma in human cells through its action on PER1. In cholangiocarcinoma, PER1 expression is decreased and periodical rhythm is lost. Cells that overexpress PER1 displayed reduced proliferation, decreased G2/M lag phase, and enhanced apoptotic induction. In vivo PER1 overexpression reduced tumor growth, proliferation, angiogenesis, and metastasis. Inhibiting miR-34a similarly promoted the metastasis of cholangiocarcinoma cells. This highlighted how manipulation of the molecular clockwork in humans may prove beneficial during the treatment of serious human diseases.

\section{Zebrafish studies}

Using miRNA sequencing technologies, pineal-enhanced and light-induced miRNAs were identified by Ben-Moshe ${ }^{60}$. One such miRNA, miR-183, was shown to downregulate e4bp4-6 which regulated the rhythmic mRNA levels of aanat2, a key enzyme in melatonin synthesis. This genome-wide approach and functional characterization of light-induced factors indicated multi-level regulation of the circadian clock by light.

Cold shock can induce acute physiological stress responses and transcriptional changes in aquatic creatures. Hung ${ }^{61}$ revealed a role for miRNAs in acute cold responses through small RNA-seq analyses, identifying numerous differentially expressed miRNAs. Overexpression of Per2 resulted in partial recovery from cold shock. Subsequently the interaction of Per 2 with miR$29 \mathrm{~b}$ was identified, and this miRNA was also found to be cold-inducible. This study characterized the functional roles of CCGs during cold tolerance

\section{Other miRNAs related to $\mathbf{C R}$}

Other model systems of note that have revealed key circadian roles of miRNA genes including the identification of miR-206 as an important regulatory factor in mammalian skeletal muscle cells ${ }^{62}$. By controlling changes of amplitude and frequency, miR-206 influences gene expression and interrupts gene synthesis. Riley ${ }^{63}$ found that miR-125a-5p affected the stability of the Lfng transcripts in the anterior mesoderm of chickens, and its inhibition disrupted CR functionality. Chickens lacking miR-125a-5p expression also displayed abnormal embryonic cell division. Nocturnin, an adenase that is expressed downstream of the CR, acts to regulate metabolism in response to biological rhythm. Nocturnin was revealed as a target of miR-122, a liver specific miR-122 known to participate in lipid metabolism ${ }^{64}$.

\section{Summary and future prospects}

Recently, miRNAs have emerged as significant players in CR timing, raising the possibility that clock-controlled miRNAs contribute to disorders of the human circadian timing system. Since the biochemical activity of an array of cell types and organs is shaped by our 24hour CR, miRNAs impart circadian modulation over a wide range of behavioral and physiological processes. Indeed, rhythmic drive regulates up to $15 \%$ of the transcriptome. Dysregulation of miRNAs, and thus the clock, is implicated in the pathogenesis of many disorders ranging from hypertension to cancer. Therefore, new therapeutic approaches targeting miRNA expression and clock physiology are of major interest. A more comprehensive understanding of the underlying molecular mechanisms that modulate miRNAs involved in $\mathrm{CR}$ and give rise to organ-specific CR transcriptomes is now required. This information will allow us to unlock the utility of miRNAs as effectors of CR physiology and in the pathophysiology of clock related disease processes.

\section{Abbreviations \\ CR, circadian rhythm; SCN, suprachiasmatic nucleus; miRNAs, microRNAs; pre-miRNA, precursor miRNA; C. elegans, Caenorhabditis elegans; ESCs, embryonic stem cells; NF, nuclear factor; CCGs, clock-controlled genes; BMP, bone morphogenetic protein; CF-2, chori- on factor 2; LPS, lipopolysaccharide}

\section{Conflict of interest}

The authors declare that they have no conflict of interest. 


\section{Ethical statement}

Not applicable.

\section{Funding}

This research was supported by Natural Science Foundation of China (No. 81873333), Natural Science Foundation of China (No. 81373683) and Zhenjiang Health and Family Planning Science Technology Key Project (No.SHW2017016).

\section{Acknowledgments}

Not applicable.

\section{References}

1 Garbazza C, Benedetti F. Genetic factors affecting seasonality, mood, and the circadian clock. Front Endocrinol (Lausanne). 2018; 9:481.

2 Mendoza-Viveros L, Bouchard-Cannon P, Hegazi S, Cheng AH, Pastore S, Cheng HM. Molecular modulators of the circadian clock: lessons from flies and mice. Cell Mol Life Sci. 2017; 74(6):1035-1059.

3 Preussner M, Heyd F. Post-transcriptional control of the mammalian circadian clock: implications for health and disease. Pflugers Arch. 2016; 468(6):983-991.

4 Takeda N, Maemura K. Circadian clock and cardiovascular disease. J Cardiol. 2011; 57(3):249-256.

5 Shetty A, Hsu JW, Manka PP, Syn WK. Role of the circadian clock in the metabolic syndrome and nonalcoholic fatty liver disease. Dig Dis Sci. 2018.

6 Shimizu I, Yoshida Y, Minamino T. A role for circadian clock in metabolic disease. Hypertens Res. 2016; 39(7):483-491.

7 Fang L, Yang Z, Zhou J, Tung JY, Hsiao CD, Wang L, Deng Y, Wang P, Wang J, Lee MH. Circadian clock gene CRY2 degradation is involved in chemoresistance of colorectal cancer. Mol Cancer Ther. 2015; 14(6):14761487.

8 Thome J, Coogan AN, Woods AG, Darie CC, Hassler F. CLOCK genes and circadian rhythmicity in Alzheimer disease. J Aging Res. 2011; 2011:383091.

9 Damulewicz M, Loboda A, Jozkowicz A, Jozef D, Elzbieta P. Interactions between the circadian clock and heme oxygenase in the retina of Drosophila melanogaster. Mol Neurobiol. 2017; 54(7):4953-4962.

10 Laranjeiro R, Whitmore D. Transcription factors involved in retinogenesis are co-opted by the circadian clock following photoreceptor differentiation. Development. 2014; 141(13):2644-2656.

11 Maiese K. Novel treatment strategies for the nervous system: circadian clock genes, non-coding RNAs, and forkhead transcription factors. Curr Neurovasc Res. 2018; 15(1):81-91.

12 Kudo T, Block GD, Colwell CS. The circadian clock gene Period1 connects the molecular Clock to neural activity in the suprachiasmatic nucleus. ASN Neuro. 2015; 7(6):e103309.

13 Mohawk JA, Green CB, Takahashi JS. Central and peripheral circadian clocks in mammals. Annu Rev Neurosc. 2012; 35(1):445-462.

14 Mermet J, Yeung J, Hurni C, Mauvoisin D, Gustafson K, Jouffe C, Nicolas D, Emmenegger Y, Gobet C, Franken P, Gachon F, Naef F. Clock-dependent chromatin topology modulates circadian transcription and behavior. Genes Dev. 2018; 32(5-6):347-358.

15 DeBruyne JP, Baggs JE, Sato TK, Hogenesch JB. Ubiquitin ligase Siah2 regulates RevErbalpha degradation and the mammalian circadian clock. Proc Natl Acad Sci U S A. 2015; 112(40):12420-12425.

16 Zhang Y, Duan C, Yang J, Chen S, Liu Q, Zhou L, Huang Z, Xu Y, Xu G. Deubiquitinating enzyme USP9X regulates cellular clock function by modulating the ubiquitination and degradation of a core circadian protein BMAL1. Biochem J. 2018; 475(8):1507-1522.

17 Lamia KA, Sachdeva UM, DiTacchio L, Williams EC, Alvarez JG, Egan DF, Vasquez DS, Juguilon H, Panda $\mathrm{S}$, Shaw RJ, Thompson CB, Evans RM. AMPK regulates the circadian clock by cryptochrome phosphorylation and degradation. Science. 2009; 326(5951):437-440. 18 Ohsaki K, Oishi K, Kozono Y, Nakayama K, Nakayama KI, Ishida $\mathrm{N}$. The role of $\{$ beta $\}-\operatorname{TrCP} 1$ and \{beta\}-TrCP2 in circadian rhythm generation by mediating degradation of clock protein PER2. J Biochem. 2008; 144(5):609-618.

19 Gnanamanickam GJE, Liewellyn-Smith LJ. Innervation of the rat uterus at estrus: a study in full-thickness, immunoperoxidase-stained whole-mount preparations. J Comp Neurol. 2011; 519(10):621-643.

20 Lee RC, Feinbaum RL, Ambros V. The C. elegans heterochronic gene lin-4 encodes small RNAs with antisense complementarity to lin-14. Cell. 1993; 75(5):843854.

21 Wightman B, Ha I, Ruvkun G. Posttranscriptional regulation of the heterochronic gene lin-14 by lin-4 mediates temporal pattern formation in C. elegans. Cell. 1993; 75(5):855-862.

22 Reinhart BJ, Slack FJ, Basson M, Pasquinelli AE, Bettinger JC, Rougvie AE, Horvitz HR, Ruvkun G. The 21-nucleotide let-7 RNA regulates developmental timing in Caenorhabditis elegans. Nature. 2000; 403(6772):901-906. 
23 Sood P, Krek A, Zavolan M, Macino G, Rajewsky N. Cell-type-specific signatures of microRNAs on target mRNA expression. Proc Natl Acad Sci U S A. 2006; 103(8):2746-2751.

24 Berezikov E. Evolution of microRNA diversity and regulation in animals. Nat Rev Genet. 2011; 12(12):846860.

25 Svobodova E, Kubikova J, Svoboda P. Production of small RNAs by mammalian Dicer. Pflugers Arch. 2016; 468(6):1089-1102.

26 Kim BM, Thier MC, Oh S, Sherwood R, Kanellopoulou C, Edenhofer F, Choi MY. MicroRNAs are indispensable for reprogramming mouse embryonic fibroblasts into induced stem cell-like cells. PLoS One. 2012; 7(6):e39239.

27 Gong Z, Wang D, Zhu S, Xia Y, Fan C, Zhao B, Jin Y.MiR-290 contributes to the low abundance of cyclin D1 protein in mouse embryonic stem cells. Acta Biochim Biophys Sin (Shanghai). 2017; 49(7):635-642.

28 Yuan K, Ai WB, Wan LY, Tan X, Wu JF. The miR290-295 cluster as multi-faceted players in mouse embryonic stem cells. Cell Biosci. 2017; 7:38.

29 Wang T, Shi SB, Sha HY. MicroRNAs in regulation of pluripotency and somatic cell reprogramming: small molecule with big impact. RNA Biol. 2013; 10(8):12551261.

30 Chen H, Wang X, Yan X, Cheng X, He X, Zheng W. LncRNA MALAT1 regulates sepsis-induced cardiac inflammation and dysfunction via interaction with miR125b and p38 MAPK/NFkappaB. Int Immunopharmacol. 2018; 55:69-76.

31 Duroux-Richard I, Roubert C, Ammari M, Presumey J, Grun JR, Haupl T, Grutzkau A, Lecellier CH, Boitez V, Codogno P, Escoubet J, Pers YM, Jorgensen C, Apparailly F.MiR-125b controls monocyte adaptation to inflammation through mitochondrial metabolism and dynamics. Blood. 2016; 128(26):3125-3136.

32 Liu Z, Chen X, Wu Q, Song J, Wang L, Li G.MiR$125 \mathrm{~b}$ inhibits goblet cell differentiation in allergic airway inflammation by targeting SPDEF. Eur J Pharmacol. 2016; 782:14-20.

33 Gao FB. Posttranscriptional control of neuronal development by microRNA networks. Trends Neurosi. 2008; 31(1):20-26.

34 Chistiakov DA, Orekhov AN, Bobryshev YV. Cardiac-specific miRNA in cardiogenesis, heart function, and cardiac pathology (with focus on myocardial infarction). J Mol Cell Cardiol. 2016; 94:107-121.

35 Liu N, Bezprozvannaya S, Williams AH, Qi X, Richardson JA, Bassel-Duby R, Olson EN.MicroRNA-133a regulates cardiomyocyte proliferation and suppresses smooth muscle gene expression in the heart. Genes Dev. 2008; 22(23):3242-3254.

36 Yi R, Poy MN, Stoffel M, Fuchs E. A skin microRNA promotes differentiation by repressing 'stemness'. Nature. 2008; 452(7184):225-229.

37 Van de Bunt M, Gaulton KJ, Parts L, Moran I, Johnson PR, Lindgren CM, Ferrer J, Gloyn AL, McCarthy MI. The miRNA profile of human pancreatic islets and beta-cells and relationship to type 2 diabetes pathogenesis. PLoS One. 2013; 8(1):e55272.

38 Liu L, Zhao X, Zhu X, Zhong Z, Xu R, Wang Z, Cao J, Hou Y. Decreased expression of miR-430 promotes the development of bladder cancer via the upregulation of CXCR7. Mol Med Rep. 2013; 8(1):140-146.

39 Shi ZM, Wang XF, Qian X, Tao T, Wang L, Chen QD, Wang XR, Cao L, Wang YY, Zhang JX, Jiang T, Kang CS, Jiang BH, Liu N, You YP. MiRNA-181b suppresses IGF-1R and functions as a tumor suppressor gene in gliomas. RNA. 2013; 19(4):552-560.

40 Bahk YC, Han E, Lee SH. Biological rhythm differences and suicidal ideation in patients with major depressive disorder. J Affect Disord. 2014; 168:294-297.

41 Chiara LM, Fred NRC, Alfredo AS, Valerio C. Retinal ganglion cells and circadian rhythms in Alzheimer's disease, Parkinson's disease, and beyon. Front Neurol. 2017; 8:162.

42 Henriques R, Mas P. Chromatin remodeling and alternative splicing: pre- and post-transcriptional regulation of the Arabidopsis circadian clock. Semin Cell Dev Biol. 2013; 24(5):399-406.

43 Brunner M, Schafmeier T. Transcriptional and post-transcriptional regulation of the circadian clock of cyanobacteria and Neurospora. Genes Dev. 2006; 20(9):1061-1074.

44 Nishii K, Yamanaka I, Yasuda M, Kiyohara YB, Kitayama Y, Kondo T, Yagita K. Rhythmic post-transcriptional regulation of the circadian clock protein mPER2 in mammalian cells: a real-time analysis. Neurosci Lett. 2006; 401(1-2):44-48.

45 Repouskou A, Prombona A.C-MYC targets the central oscillator gene Per1 and is regulated by the circadian clock at the post-transcriptional level. Biochim Biophys Acta. 2016; 1859(4):541-552.

46 Garaulet DL, Sun K, Li W, Wen J, Panzarino AM, O'Neil JL, Hiesinger PR, Young MW, Lai EC.MiR-124 regulates diverse aspects of rhythmic behavior in drosophila. J Neurosci. 2016; 36(12):3414-3421.

47 Chen X, Rosbash M.Mir-276a strengthens Drosophila circadian rhythms by regulating timeless expression. Proc Natl Acad Sci U S A. 2016; 113(21):E2965-2972. 
48 Sun K, Jee D, de Navas LF, Duan H, Lai EC. Multiple in vivo biological processes are mediated by functionally redundant activities of drosophila mir-279 and mir-996. PLoS Genet. 2015; 11(6):e1005245.

49 Paola C, Alberto B, Federica S, Gabriella MM, Claudia T, Cristiano DP, Gabriella C. Modulation of miR210 alters phasing of circadian locomotor activity and impairs projections of PDF clock neurons in Drosophila melanogaster. PLoS Genet. 2018; 14(7):e1007500-.

50 You S, Fulga TA, Van VD, Jackson FR. Regulation of circadian behavior by astroglial microRNAs in Drosophila. Genetics. 2018; 208(3):1195-1207.

51 Cheng HY, Papp JW, Varlamova O, Dziema H, Russell B, Curfman JP, Nakazawa T, Shimizu K, Okamura H, Impey S, Obrietan K.MicroRNA modulation of circadian-clock period and entrainment. Neuron. 2007; 54(5):813-829.

52 Shende VR, Neuendorff N, Earnest DJ. Role of miR-142-3p in the post-transcriptional regulation of the clock gene Bmal1 in the mouse SCN. PLoS One. 2013; 8(6):e65300.

53 Ding X, Sun B, Huang J, Xu L, Pan J, Fang C, Tao Y, Hu S, Li R, Han X, Miao P, Wang Y, Yu J, Feng X. The role of miR-182 in regulating pineal CLOCK expression after hypoxia-ischemia brain injury in neonatal rats. Neurosci Lett. 2015; 591:75-80.

54 Zhang W, Wang P, Chen S, Zhang Z, Liang T, Liu C. Rhythmic expression of miR-27b-3p targets the clock gene Bmal1 at the posttranscriptional level in the mouse liver. FASEB J. 2016; 30(6):2151-2160.

55 Gao Q, Zhou L, Yang SY, Cao JM. A novel role of microRNA 17-5p in the modulation of circadian rhythm. Sci Rep. 2016; 6:30070.

56 Kiessling S, Ucar A, Chowdhury K, Oster H, Eichele G. Genetic background-dependent effects of murine micro RNAs on circadian clock function. PLoS One. 2017; 12(4):e0176547.
57 Yoo SH, Kojima S, Shimomura K, Koike N, Buhr ED, Furukawa T, Ko CH, Gloston G, Ayoub C, Nohara K, Reyes BA, Tsuchiya Y, Yoo OJ, Yagita K, Lee C, Chen Z, Yamazaki S, Green CB, Takahashi JS. Period2 3'-UTR and microRNA-24 regulate circadian rhythms by repressing PERIOD2 protein accumulation. $P$ NATL A SCI. 2017; 114(42):201706611.

58 Li A, Lin X, Tan X, Yin B, Han W, Zhao J, Yuan J, Qiang B, Peng X. Circadian gene Clock contributes to cell proliferation and migration of glioma and is directly regulated by tumor-suppressive miR-124. FEBS Lett. 2013; 587(15):2455-2460.

59 Han Y, Meng F, Venter J, Wu N, Wan Y, Standeford $\mathrm{H}$, Francis H, Meininger C, Greene J, Jr., Trzeciakowski JP, Ehrlich L, Glaser S, Alpini G.MiR-34a-dependent overexpression of Per1 decreases cholangiocarcinoma growth. J Hepatol. 2016; 64(6):1295-1304.

60 Ben-Moshe Z, Alon S, Mracek P, Faigenbloom L, Tovin A, Vatine GD, Eisenberg E, Foulkes NS, Gothilf Y. The light-induced transcriptome of the zebrafish pineal gland reveals complex regulation of the circadian clockwork by light. Nucleic Acids Res. 2014; 42(6):37503767.

61 Hung IC, Hsiao YC, Sun HS, Chen TM, Lee SJ. MicroRNAs regulate gene plasticity during cold shock in zebrafish larvae. BMC Genomics. 2016; $17(1): 922$.

62 Zhou W, Li Y, Wang X, Wu L, Wang Y. MiR-206-mediated dynamic mechanism of the mammalian circadian clock. BMC Syst Biol. 2011; 5:141.

63 Riley MF, Bochter MS, Wahi K, Nuovo GJ, Cole SE. Mir-125a-5p-mediated regulation of Lfng is essential for the avian segmentation clock. Dev Cell. 2013; 24(5):554-561.

64 Green CB, Douris N, Kojima S, Strayer CA, Fogerty J, Lourim D, Keller SR, Besharse JC. Loss of Nocturnin, a circadian deadenylase, confers resistance to hepatic steatosis and diet-induced obesity. Proc Natl Acad Sci U S A. 2007; 104(23):9888-9893. 